\title{
Wave-based motion and slewing control of a double-appendage, flexible system with ungrounded actuator through development of direct actuator force control
}

\author{
Hossein Habibi ${ }^{\text {a*}}$, William O'Connor ${ }^{\text {b }}$ \\ ${ }^{\text {a }}$ School of Science, Engineering and Design, Teesside University, Middlesbrough TS1 3BX, United Kingdom \\ ${ }^{\mathrm{b}}$ UCD School of Mechanical and Materials Engineering, UCD Belfield, Dublin 4, Ireland
}

\begin{abstract}
Wave-based control is a relatively new approach which has already been applied successfully to control a range of underactuated, flexible mechanical systems, such as robots and cranes, through a rest-to-rest manoeuvre after it identifies, then measures and finally exploits the propagation time delay effects inherent in flexible systems. In this technique, the actuator motion is directly controlled in a way that, simultaneously, indirectly controls the motion of the attached flexible systems, thereby combining position control and active vibration damping. A significant development of this strategy is here presented, in which the directly controlled actuation variable is force (or torque) rather than position or motion, as before. This new formulation is particularly relevant for motion control of systems whose actuators are not grounded, such as spacecraft, with thrusters, reaction wheels or magnetic torquers, where the natural, actuator input variable is a force or torque, to be specified by the control law (rather than actuator motion). This development considers a real (non-ideal) actuator with significant inertia and thus associated time delay in responding to input signals. The new control design approach is presented, and applied to planar, translation and rotation (slewing) of an approximate model of a spacecraft having two flexible appendages, representing for example, solar panel arrays or antennas, modelled as systems of lumped masses and springs, with (possibly) different appendages on one spacecraft. Despite the dynamic complexity of the multiple attached flexible arrays, having many degrees of freedom, with complex vibration modes, and use of a non-ideal, ungrounded actuator, the proposed control strategy can achieve precise motion control, whether translation, rotation or both, while actively suppressing vibrations of the flexible appendages.
\end{abstract}

Keywords: Wave based control, flexible appendages, actuator force control, spacecraft control.

\section{Introduction}

Wave-Based Control (WBC) approaches the problem of controlling under-actuated, flexible systems by considering the interaction between the actuator and the flexible system as one of launching and absorbing of mechanical "waves" (propagating disturbances). The wave absorption idea had been used before, for active damping of sound and vibration [1,

2]. The novelty was to combine position control and active vibration damping in a single actuator, by simultaneously

\footnotetext{
*Corresponding author: e-mails: H.Habibi@tees.ac.uk; hossein.habibi@ucdconnect.ie.
} 
launching and absorbing motion in a controlled way which was first proposed by O'Connor and Lang [3]. They found that the process of absorbing a wave which had been previously launched into a system of lumped masses, moved the system twice the steady-state (or net, or DC) displacement associated with the original input wave. To this end, they defined a wave transfer function (WTF), or $G(\mathrm{~s})$, worked in the Laplace complex frequency domain, that relates the motion of one mass to that of its neighbour on one side in a flexible string of lumped masses. The most important features of the WTF are that: 1) they cause an approaching wave motion to be absorbed (and so passed on to the next WTF) without imposing a steady-state constraint; 2) the steady state gain in this process is unity; and 3) there is an associated time delay, or phase lag, or transient, which is approximately second order, underdamped. The WTF $G(\mathrm{~s})$ is not easy to implement in the time domain, involving irrational functions of $s$ in the Laplace domain. However it can be approximated by

$$
G(s) \cong \frac{\omega_{n}^{2}}{s^{2}+\omega_{n} s+\omega_{n}^{2}}
$$

with $\omega_{n}=\sqrt{k / m}$, or, in the time domain, by an analog system, composed of a spring, $k$, a mass, $m$, and a grounded viscous damper, with damping coefficient $c=\sqrt{k / m}$ [4]. Using this principle, a control scheme was designed which removed vibrations and at the same time re-positioned the system accurately.

This technique has many advantages, solving most of the difficulties associated with other approaches. In summary, it does not require a good system model, and so is robust to modelling errors and system changes. It collocates sensing and actuation that avoids the measuring error due to the time delay associated with the flexibility of mechanical system, greatly helping stability. It does not need sensors throughout the flexible system but does all the sensing where the actuator meets the flexible system. At this interface only two variables are sensed, and this sensing is easily achieved. As well known, variations on classical controllers, such as fractional order controllers, remain blind to the peculiar dynamic features and non-minimum phase behaviour which characterise oscillatory systems, especially because of time delays between actuator motion and tip motion. By contrast, WBC, through a rest-to-rest manoeuvres in times, first identifies, then measures and finally exploits the propagation time delay effects inherent in flexible systems, but without relying on a full system model, achieving close to the theoretical minimum time of time-optimal control [5] in a robust and generic way. Further research on extensions to or applications of WBC has been published in, for example, [6-11]. Much of this work was tested using computer simulation and numerical models, but experimental work has also been carried out and reported on a variety of test rigs to verify the validity of the method practically [4, 12-13]. For example, the ideas were applied to a very light and flexible arm, driven by a DC motor, to re-position a tip mass, supported on an almost frictionless air table, to a target position in a horizontal plane [4]. Likewise, the idea of WBC has been successfully applied on cable driven manipulators and the cable structures working within large workspaces that are very flexible and challenging to be controlled [13-15]. 
A prototypical under-actuated flexible system is a rectilinear string of masses and springs controlled by a linear actuator, as shown in Fig.1 (here with just two masses and springs). In the methodology of WBC as presented in the literature [310], the flexible control system is controlled by directly controlling the motion of an actuator, $x_{0}$, to control, indirectly, the motion of the attached flexible system, to combine position control and active vibration damping. When the actuator moves, its motion, $x_{0}$, can be considered to have two physical effects on the attached flexible system at every instant. a) It is launching a disturbance wave into the flexible system named 'launch wave' denoted by $a(\mathrm{t})$, and $\mathrm{b}$ ) it is responding to a returning disturbance wave, either to reflect it back into the system, or to absorb it, or (more likely) to bring about some instantaneous combination of reflection and absorption. These two effects, launching and absorbing/reflecting, at every instant, will be associated with two notional components, $a(t)$ and $b(t)$ respectively, of the actuator's motion, $x_{0}(t)$, where

$$
x_{0}(t)=a(t)+b(t)
$$

Under the WBC strategy, the controller specifies the launch component of the actuator's motion, usually setting it to half the input motion reference, or $1 / 2 r(t)$. To this it adds a measured 'return wave', denoted $b(t)$, as a special kind of positive feedback, to form the combined input request, $c(\mathrm{t})$, to the actuator controller. Therefore, the controlled input, $c(t)$, to the actuator becomes

$$
c(t)=1 / 2 r(t)+b(t)
$$

This implementation is shown in Fig.1. If the actuator were ideal, with unity gain, $x_{0}(t)$ would be identical to $c(t)$, implying $1 / 2 r(\mathrm{t})=a(\mathrm{t})$, the actuator's launch component, for all $t$. (Compare Eqs.(2) and (3)). For a real actuator with unity steady-state gain, the equality may become exact only at steady-state when $b(\infty)=1 / 2 \mathrm{r}(\infty)=a(\infty)$.

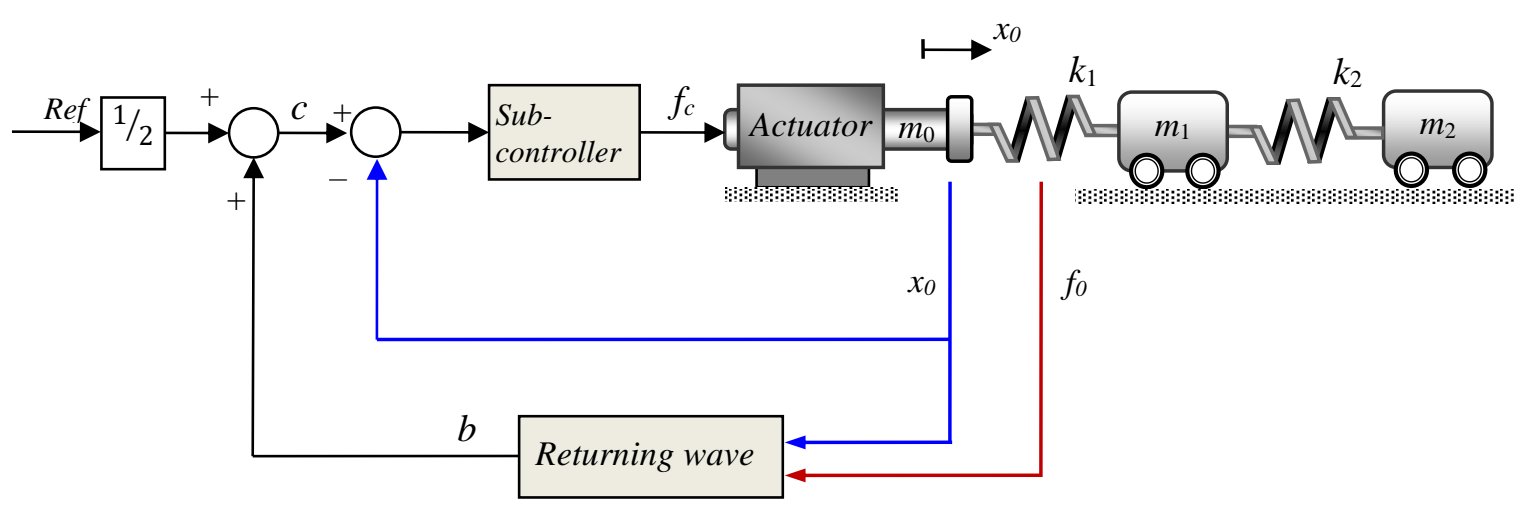

Fig. 1. Wave-based control of a 2-DOF mass-spring system using a single, non-ideal actuator and its sub-controller.

In fact, the returning or absorbing wave, $b(\mathrm{t})$, is a delayed, more dispersed, version of the launch wave that can be defined and measured in various ways. This will be explained in Section 2.2. The slightly different resulting control schemes produce similar control responses, so to some extent the choice is a matter of convenience [16]. In this work as shown in 
Fig. 1, the scheme is based on a time integral of the interface force and on a fixed value of mechanical or wave impedance. It will be referred to as the 'force impedance' method. In effect, this causes the actuator to behave like a viscous damper to the returning motion, actively absorbing the system vibrations without need for inclusion of damping elements, analogous to a matched impedance at the end of an electrical transmission line. This is also what makes the controller so stable. The returning wave is determined from two independent, interface measurements; the actuator position, $x_{0}(\mathrm{t})$, and the force, $f_{0}(\mathrm{t})$, which the actuator applies to the flexible system. Further details on the 'force impedance' approach to measure returning waves can be found in [16-18]. Note in passing, however, that for rest-to-rest motion, conservation of momentum implies the force integral will become zero, so that at steady-state, the final value of $b$ will be $1 / 2 x_{o}$, which will supply the second half of the reference displacement on settling.

This simple approach for a rectilinear system, such as in Fig.1, works well not just for the 1-D translational motion depicted, but also for the control of more complex systems, with 2-D and 3-D dynamics, undergoing translation and rotation, with up to 6 degrees of freedom in the actuator, and arbitrarily large degrees of freedom in the attached flexible system. References $[8,9,12$, and 18$]$ describe some of this work, extending WBC to very complex systems. In all cases, any motion of the actuator launches a wave into the system, which sooner or later comes back to the actuator, where it is absorbed. No detailed model of the system is needed. All the measuring is done at the actuator.

In WBC, changes in the system require no or only little adjustment of the control arrangement and cause relatively small changes in the general shape of the component waveforms. For example, as the system order grows or as the system becomes more flexible, the main change is simply an increase in the time delay between the launch wave's reaching the half target position and the return wave's attaining the same dc value. The returning wave carries the information needed to absorb the vibrations. If the system changes, the returning wave changes and the controller adapts, in real time. If for any reason the absorption is not perfect, say $90 \%$ (for example because of sensing errors, wave measurement errors, or nonideal actuator performance) $10 \%$ of the returning wave is then reflected back into the system, and then $90 \%$ of this $10 \%$ will be absorbed on its return to the actuator. In this way vibratory motion is quickly absorbed, even with a less-than-perfect implementation. All these features of WBC have been discussed in the literature. They are mentioned here to provide more context for what follows.

The wave-based control strategy determines $c$, the desired actuator motion, as discussed through Eqs. (2) and (3). As discussed in previous work on WBC [16-18], there is no need to assume an ideal actuator response, provided that the actuator has its own position sub-controller, to respond to the input $c$. WBC fulfills its control task when the actuator's subcontroller manages to make $x_{0}$ follow $c$ as well as it can, attempting to make $x_{0}=c$, which would apply exactly only if the actuator were ideal. Observe that this standard WBC arrangement therefore has motion controlling motion (Fig. 1), that is, 
the actuator's own motion is controlling the system's motion, in response to a motion reference input. Obviously, forces are needed (or torques in the equivalent rotational system) for motion to happen, shown as $f_{c}$. The point, however, is that the implementation of WBC does not get involved in specifying these forces, as the details of producing and controlling the forces are the responsibility of the actuator sub-controller, typically using a local feedback of the actuator motion (not shown). This motion sub-control system could, for example, be an off-the-shelf, commercial, motion control system, whose control law is unknown to the design engineer. WBC will still work well (provided only that the actuator and sub-controller have a minimum dynamic range and bandwidth).

This actuator, black-box approach, in which WBC does not concern itself with forces, is well suited to situations where the actuator is grounded, such as in a robotic arm on a base, or with a crane on a fixed structure. The ground supplies a resistance to the actuator's reaction forces, whether pushing or pulling the flexible system. By contrast, it is sometimes preferable, or necessary, to have WBC specify directly (that is, control) the force (or torque) to act on the actuator, and so on the system. This is the topic of the present work.

Imagine the flexible system in Fig.1 to be floating in space, with the actuator replaced by a thruster, for example (removing therefore both the grounding and the sub-controller). The control system then needs to specify the force, $f_{c}$, directly, which must move the actuator and attached flexible system in a controlled way. With force now controlling motion, this is closer to the way most engineers think of motion control.

Figure 2 represents schematically an important situation where this challenge arises. It represents a satellite body which has flexible appendages, such as solar panel arrays, instrument booms, large radiation shields or antennae. To facilitate the deployment of such appendages, their structures frequently incorporate sprung hinges of various kinds, often between more rigid elements, such as solar panels. They can be very large. Their natural frequencies, therefore, can be low, and are easily excited, for example by the regular manoeuvres necessary to correct the attitude as it drifts over time due to small external disturbances, or for orbital corrections. Natural vibration damping is often very light. In such systems, the actuators, such as thrusters, reaction wheels, or magnetic torquers, will be on board the main satellite body. When the satellite needs to make an attitude (pointing direction) adjustment or a change in translational speed, perhaps for orbital correction, then the control system must specify suitable forces and torques in the actuators, to produce $f_{c}$ and $M_{c}$, which then move the system while, ideally, also controlling the vibration of the flexible appendages. The vibration control aspect is important not just for structural reasons, but also to minimize the waiting time before, for example, scientific observations can recommence or communication channels recover full capacity having been impeded by the lingering vibrations. 


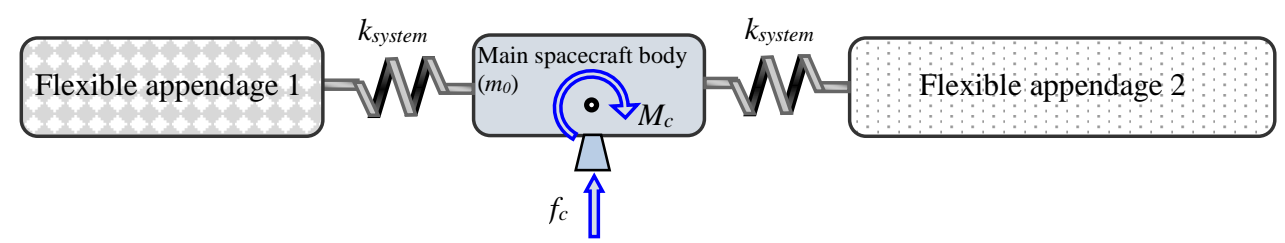

Fig.2. Schematic representation of a spacecraft model with different flexible appendages.

To recover the advantages of WBC, a first thought might be to design a sub-controller for this case, to get back closer to Fig.1. One might then think of the satellite main body as equivalent to the motion actuator of Fig.1, controlling the flexible appendages, as well as itself. This option was explored, as described below. But a series of problems emerged. First of all it is not obvious what kind of sub-controller should be used for actuator (satellite main body) position control under force input. If a classical PID controller is used, it is not clear how it should be tuned. Should the tuning be done with or without the flexible load attached? Should it be done with the higher level control system (WBC) in operation or not? When is the resulting control system optimal? More seriously, it was found that inappropriate tuning could make the entire system unstable, as illustrated below. For these reasons, a way was sought to avoid introducing the sub-controller, and to use WBC to control the actuator force (or torque) directly, especially for cases such as satellite motion control where this was demanded by the problem.

In the following, in Section 2, the adaptation of WBC for force controlled actuators and its new formulation is presnted and discussed, and is then tested on a simple floating system. Section 3 details the application of the developed version of WBC to the case of motion control of a spacecraft model with large flexible appendages. Section 4, presents and discusses the modelling results on the performance of the adapted approach applied on the modelled spacecraft for different maneuvers. Finally, summary and concluding remarks are given in Section 5.

\section{Force controlled actuators in WBC}

\subsection{Using a sub-controller, acting as a non-ideal actuator}

As discussed, the first approach investigated was to adapt the "traditional" WBC, represented in Fig.1, to suit cases with floating actuators, represented by Fig. 2. As has been explained, in cases such as Fig.1, the actuator is supplied with its own sub-controller, with motion request input $c(t)$, leaving this sub-controller with the task of deciding the actuator force to achieve the requested motion. The wave-based control is then happening at a higher (supervisory) level. Thus WBC and the sub-control system work independently, with no attempt to communicate or coordinate.

One of the standard ways of controlling an actuator is closed-loop control with the ubiquitous PID error feedback, involving three parameters. The parameters can be interpreted from a time perspective: the $P$ (proportional) term depends on the present error, the $I$ (integral) term on the accumulation of past errors, and the $D$ (differential) term is a prediction of 
future errors based on current rate of change of the error [19]. These three terms are combined in a weighted sum, with weights typically determined by experience-based rules, trading off desirable features in the time response, such as transient time, overshoot and settling time. But this classical approach does not work well for the current problem. Even for a simple rectilinear system such as in Fig.1, there is no explicit and definite method to adjust the trade-off between the constants [20]. The three PID constants need to be adjusted when even small changes occur in either the model or the reference input. The tuning challenge even becomes more challenging than usual when the actuator has significant inertia in comparison with other system components, which is typical of the satellite problem.

Besides the problem of PID tuning, when the system shown in Fig. 1 was tested for different values of actuator mass, an instability difficulty emerged as the mass was increased. This was found even in a relatively simple 1-D rectilinear case, raising concerns that the problem would be worse in more complex systems, such as the 2-D satellite model shown in Fig.2 which will be discussed in Section 3. Increasing the mass of the actuator can also be considered as equivalent to having an actuator which is less ideal, having a poorer dynamic response to requested inputs. To illustrate the situation, a set of values of $m_{1}=m_{2}=1 \mathrm{~kg}$ and $k_{1}=k_{2}=10 \mathrm{~N} / \mathrm{m}$ is given to the uniform, damping-free, rectilinear flexible system shown in Fig. 1 with a ramp input up to $1 \mathrm{~m}$ displacement. The PID sub-controller was first tuned in a normal way for an optimum performance on its own. It was then incorporated into the WBC system. The response was found to be greatly influenced by the mass of actuator, $m_{0}$. Figure 3 shows the instability in the response, which becomes worse as the actuator mass increases.

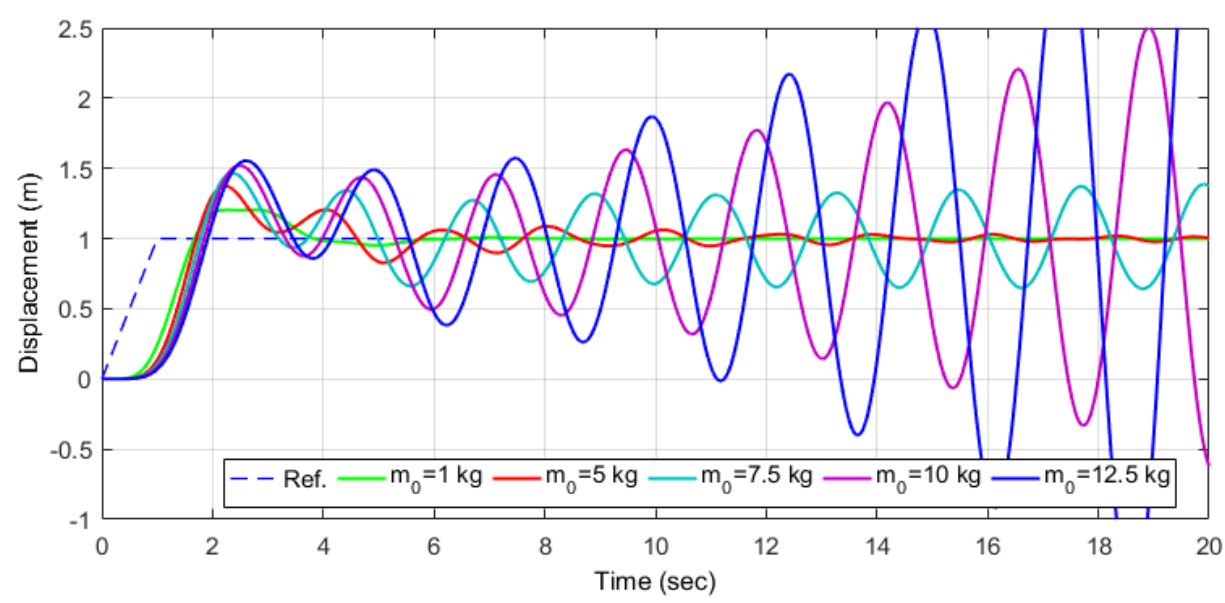

Fig. 3. Increasing instability of the two-mass flexible system with increasing actuator mass $\left(m_{0}\right)$ using standard WBC and PID tuned sub-controller.

It is seen that, when the actuator has roughly the same mass as the system masses $\left(m_{0}=1 \mathrm{~kg}\right)$, the control system behaves normally for $\mathrm{WBC}$, reaching the target and quickly coming to rest. As the actuator mass becomes larger, however, apparently it becomes more difficult for the actuator to respond to the information coming back from the flexible system, and so to absorb the vibrations. In other words, the heavy actuator acts as a large inertia in the series of lumped masses, with a strong dynamic discontinuity. While the returning wave, according to Fig. 1 , is based on both $x_{0}$ and $f_{0}$, that is, the actuator motion and the force on the flexible system, it becomes harder for the effects of $f_{0}$ to be taken into account. 
Meanwhile the PID controller takes direct care only of the actuator, and takes little or no account of what is happening beyond it. So the flexible control aspects deteriorate, to the point of instability.

Alternative strategies for the sub-controller proved no better, as the sub-controller and the wave-based control combined poorly, and even seemed to be in opposition to each other. Overall, as expected, it was observed that generic feedback control strategies, such as PID control, do not work well, partly because they fail to take account of the non-minimum phase and time delay effects that characterise flexible systems. A more radical re-think was required if the benefits of WBC were to be retained. Rather than try to establish some kind of rapport between WBC and the actuator sub-controller, a way was sought to eliminate the sub-controller completely and to use WBC to control directly the force acting on the actuator, while still working on position control and active vibration damping.

\subsection{Adapting WBC to directly control input force}

To eliminate the actuator sub-controller, a way was needed to convert the overall reference motion input, $r(t)$, into a force which would cause the actuator to achieve the two tasks (position control and active vibration damping), simultaneously. Eventually an arrangement was developed to do just that. The idea is shown in Fig. 4, for the case of control of a rectilinear mass-spring (1-D motion). The actuator mass is now considered to be part of the mass-spring string of the flexible system. It is taken as the last mass, $m_{0}$, say on the left hand end. To the left of that $m_{0}$ mass, the system is imagined to be extended further, by adding one more notional spring which in turn is attached to a notional, ideal, position actuator.

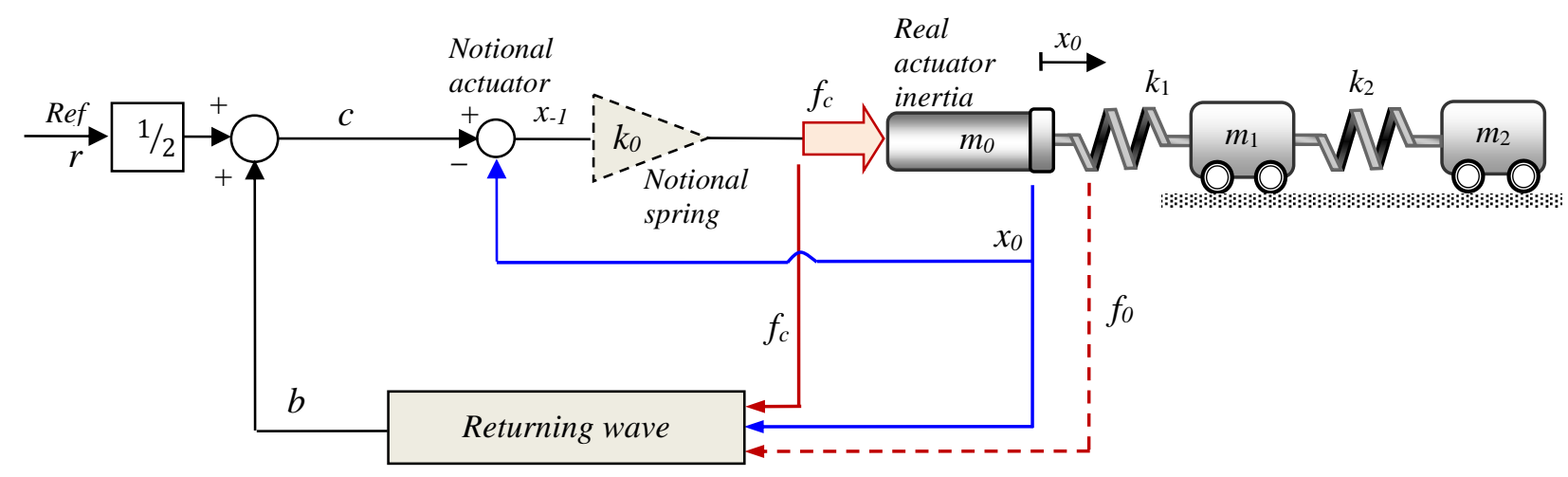

Fig. 4. The developed scheme in WBC strategy to act through direct actuator force control

To clarify the developed idea further, a theoretical background is presented concisely as follows.

To identify $a(\mathrm{t})$ and $b(\mathrm{t})$ in the physical system, three definitions, or methods of establishing, have been presented in the WBC literature. The first approach is expressed in terms of the WTF $G(\mathrm{~s})$. The s-domain versions of $a(\mathrm{t})$ and $b(\mathrm{t})$ i.e. ( $A$ and $B$, respectively) are given by 


$$
\begin{gathered}
A=X_{0} \frac{1}{1-G^{2}}-X_{1} \frac{G}{1-G^{2}} \\
B=X_{0}-A
\end{gathered}
$$

where $G$ is defined by Eq. (1), and $X_{1}$ is the s-domain version of $x_{1}$ (the displacement of the first lumped mass, $m_{1}$ ) as indicated in Figs. 1 and 4. While this first formulation is conjectured to be the "best" (at least for long, uniform systems), it gives rise to practical problems. It is very challenging to get the required time-domain values $a(\mathrm{t})$ and $b(\mathrm{t})$ from these equations as they stand. Although this problem has been solved in [3], but it is computationally expensive and slow.

A more practical approach involves reformulating Eq. (4) as

$$
A=X_{0}-G X_{1}+G^{2} A=X_{0}-G\left(X_{1}-G A\right)
$$

with $B$ from Eq. (5) as before. The required variable $A$ is now implicit in Eq. (6) (appearing on both sides). But because the transfer function $G$ on the right-hand side has zero instantaneous response, the time implementation of this equation works well when incorporated into a classical control arrangement in block diagram form, as presented in WBC literature [4]. Nevertheless, it should be noted that the WTF $G$ is an approximation of the function that relates the motion of one mass to that of its neighbour and it is not easy to be implemented exactly.

The third approach, computationally the least demanding, determines $a(\mathrm{t})$ and $b(\mathrm{t})$ simply as (in each case) either

$$
\begin{aligned}
& a(t)=\frac{1}{2}\left(x_{0}(t)+\int_{0}^{t} \frac{f(t)}{Z} d t\right)=\frac{1}{2}\left(x_{0}(t)+\omega_{n} \int_{0}^{t}\left(x_{0}-x_{1}\right) d t\right) \\
& b(t)=\frac{1}{2}\left(x_{0}(t)-\int_{0}^{t} \frac{f(t)}{Z} d t\right)=\frac{1}{2}\left(x_{0}(t)-\omega_{n} \int_{0}^{t}\left(x_{0}-x_{1}\right) d t\right)
\end{aligned}
$$

where $f(\mathrm{t})$ is the force at the actuator, or $f_{0}$ (as indicated in Figs. 1 and 4), acting in the first spring, $\omega_{n}=\sqrt{k / m}$, and $Z$ is an impedance value of $\sqrt{k_{1} m_{1}}$, corresponding to the first spring stiffness, $k_{1}$, and first mass, $m_{1}$ [4]. This option meets the control implementation requirements and works surprisingly very well when incorporated into the control systems. Depending on which of the two forms is used, the second measured variable could be force, $f_{0}$, or position $x_{1}$. In this work as will be discussed in the following, the developed scheme is based on the last option (Eqs. 7 and 8) to work out the returning wave, $b(\mathrm{t})$, due to its simplicity, generality to be used for various applications, and also presence of force quantity in the approach.

One main feature of $b(\mathrm{t})$ is that it will always lag any assigned value of $a(\mathrm{t})$ by a finite time. This can be seen, for example, by assuming a jump in the acceleration of $x_{0}$ which can be shown to leave $b$ instantaneously unchanged. The time delay is always significant, and, the longer and more flexible the system, the greater it is. Thus the controller has no practical difficulty in setting the input to the actuator to be the sum of $a+b$, because it can change $a$ as rapidly as desired (and therefore 
$x_{0}$ ) without having instantaneously to change $b$ as well. Also, initially $x_{0}$ is simply $a$. Note as the system becomes more and more rigid, the time delay between $b$ and $a$ eventually becomes negligible. This interpretation is clearest when $a$ and $b$ are defined by Eqs. (7) and (8) with $k_{1}=\infty=Z$, or $x_{0}=x_{1}$ for all $t$.

The interaction between $a$ and $b$ can be summarised through stating that, $a$, pushes the system. In the process of simultaneously adding in $b$ (the system response which is delayed with respect to $a$ ) the system, through the controller, pulls the actuator. But it does so "gently", with just the right amount of "give" to dampen or absorb the vibrations actively.

In the diagram depicted in Fig. 4, the input to this ideal, notional actuator is $c$. As before, $c$ is set to be half the reference input, $r$, plus the returning displacement wave, $b$. This notional actuator pushes the system through the notional spring, of stiffness $k_{0}$. The whole purpose of this notional arrangement is to determine the control force, $f_{\mathrm{c}}$, to be applied to the actuator mass. This corresponds to the force in this notional spring, so it is equal to the stiffness times the spring compression, or

$$
f_{c}=k_{0}\left(c-x_{0}\right)
$$

The estimation of the returning displacement wave, $b$, requires a modification from the previous arrangement presented by Eq. (8). As before, $b$ is defined as the component of the actuator motion corresponding to the returning wave, the part which should be absorbed. Also as before, to determine $b$ at least two independent variables need to be measured. But instead of measuring them where the actuator meets the flexible system, that is after (or to the right of) the actuator mass, they are now measured before (to the left), where the controlled force is applied. So the two variables for wave measurement are the applied control force, $f_{\mathrm{c}}$, and the actual, measured, actuator motion, $x_{0}$. Then the force impedance expression is reformulated as

$$
b=\frac{1}{2}\left(x_{0}-\frac{1}{Z_{c}} \int_{0}^{t} f_{c}(t) d t\right)
$$

The term $Z_{c}$ is a mechanical impedance, with dimensions of force (or torque) over velocity (linear or angular). Its value is not critical, but is of the order of $\sqrt{ }(k . m)$, where $k$ and $m$ are representative values of the lumped stiffness and mass values on the system model. The precise value can be used to fine tune the response to suit a particular application, for example trading off between rise time, overshoot and settling time. The value of the notional spring, $k_{0}$, can also be chosen to adjust the response. A higher value implies a stiffer system, with larger forces and accelerations, whereas a lower value gives a more gentle response with reduced forces. (The value can also be seen as a simple gain term at the relevant point in the forward control loop, but this view is less informative about its role.)

Figure 4, shows a possible third input to the determination of $b$, namely $f_{o}$, the interface force between the actuator mass and the rest of the flexible system. The idea was that, especially when the actuator mass was large, the vibrations of the 
flexible system would have a small impact on the motion of $x_{0}$, and so this information flow would be diminished. So the measured force $f_{o}$ could be used to try to help the controller do a better job on vibration absorption. The expression for $b$ then became

$$
b=\frac{1}{2}\left(x_{0}-\frac{1}{Z_{c}} \int_{0}^{t} f_{c}(t) d t+\frac{1}{Z_{0}} \int_{0}^{t} f_{0}(t) d t\right)
$$

using three measurements, $f_{c}, f_{o}$ and $x_{\mathrm{o}}$. This new WBC formulation directly determines the force to be applied to the actuator and so, indirectly, to the flexible system. If this force is supplied by, say, an electric motor, then the mass $m_{0}$ in Fig. 4 can be considered as the rotor inertia, now considered as the beginning of the flexible system, on which the magnetic force acts directly. Then, the attached masses, $m_{1}$ and $m_{2}$, might represent, say, a multibody, flexible robot arm. For rotational systems, torque replaces force and angular displacement (rotation) replaces linear displacement, but otherwise the analysis is the same. In the case of a satellite, for example, with flexible appendages, as shown in Fig. 2, the main satellite body corresponds to the "actuator", of mass $m_{0}$, and the control force or moment $\left(f_{c}, M_{c}\right)$ is then supplied by thruster jets or reaction wheels. The flexible appendages would correspond to the attached springs and masses. This is clearly a floating, or ungrounded system, with WBC now taking care of specifying the forces or torques for position control.

\subsection{Verification example: Performance of Non-ideal actuator associated with time delay under new WBC development}

To illustrate the application of this new version of WBC, a simple, rectilinear, floating structure with a non-ideal actuator of $m_{0}=50 \mathrm{~kg}$ as in Fig. 4 was used, with $m_{1}=m_{2}=1 \mathrm{~kg}$ and $k_{1}=k_{2}=10 \mathrm{~N} / \mathrm{m}$. Note that the mass ratio $\left(m_{0} / m_{1}\right)$ is therefore 50 whereas, according to Fig. 3, the same system using a PID sub-controller began to be unstable for ratios above 5. So the actuator here is associated with a high level of non-ideality which causes considerable time delay in the response of the system compared to that of an ideal actuator as shown in Fig.5. The force input is here applied to the real actuator, $m_{0}$. The aim is to move this system 1 meter from rest to rest. The response is shown in Fig. 5, for a wide range of values of the stiffness $k_{0}$ of the notional spring used in the model of non-ideal actuator. It also presents the time response of an ideal actuator (shown by dash line) for comparison. The system is now very stable, and settles down quickly, precisely at target, for all values of $k_{0}$. As can be seen the choice of $k_{0}$ provides a classical trade-off between rise-time, overshoot, and settling time. Even in the slowest case $\left(k_{0}=10 \mathrm{~N} / \mathrm{m}\right)$, although it brings about a considerable delayed response but it indicates that a low input force is managing to move a heavy actuator and control a very light system beyond it, in a good control performance to target. It is also observable that as the stiffness of notional spring $\left(k_{0}\right)$ increases, as expected, the time delay becomes smaller and the behaviour of the system approaches to that of an ideal actuator. 


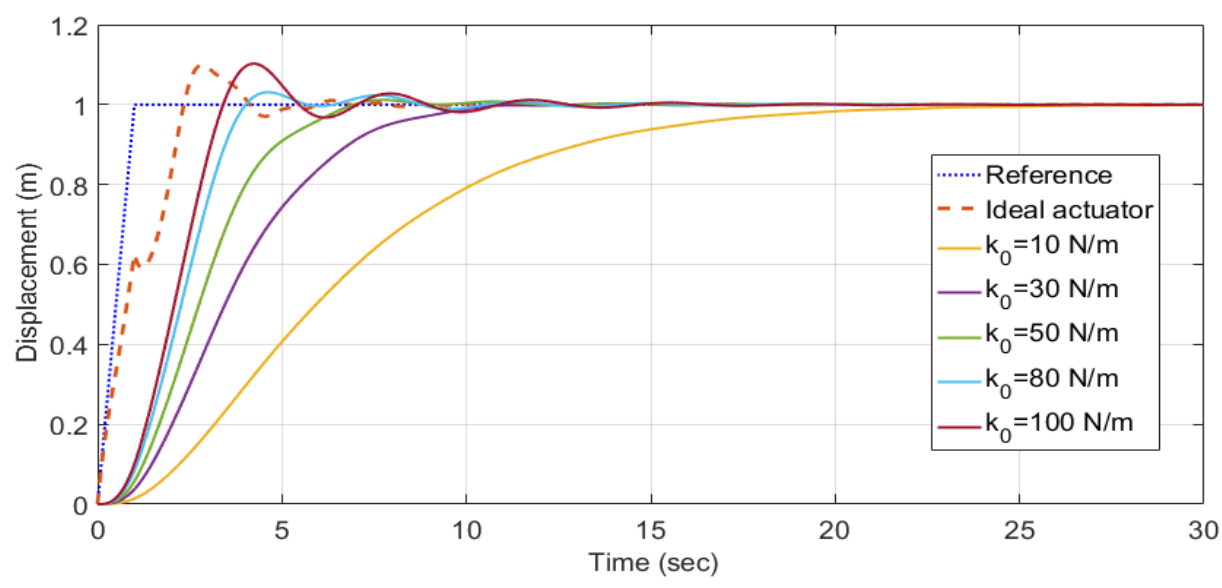

Fig.5. Responses of the real actuator applied to control the system shown in Fig. 4 for different values of $k_{0}$ (or input force, $f_{c}$ ) using the new WBC formulation compared with that of an ideal actuator (shown by dash line)

Therefore, it can be seen that the actuator does not have to be good enough to be approximated by unity. The main requirement is that the steady-state gain be unity. But the actuator can have its own dynamics and inertia, which can behave with time delay and far from ideal. To make this point clearer, the rectilinear system above was tested at a constant stiffness $\left(k_{0}=10 \mathrm{~N} / \mathrm{m}\right)$ with a range of values of the actuator mass $m_{0}$ that is the parameter dominating the inertia of the non-ideal actuator. The response of the actuators is shown in Fig. 6 for these values where the time response of an ideal actuator (as the most responsive performance) has also been depicted for comparison. As it can be seen, the more massive the actuator is, the more time delay it builds in response to the actuator input. Nevertheless, even when the mass ratio is too high $\left(m_{0}=100 \mathrm{~kg}\right)$, the controller performance does not lead to a steady-state error or instability. On the other hand, it is observed that in the best and fastest response $\left(m_{0}=1 \mathrm{~kg}\right)$, despite the considerable time delay in its rise time compared to the ideal response, it has approximately a same settling time as that of the ideal actuator. Overall, it can be inferred that the performance characteristics of the new configuration of controller, apart from slight time delay in response, are not affected by the dynamics of actuator. The only need is that, for good responses, the actuator bandwidth should extend approximately to the highest frequencies present in the system which need to be controlled, but even this is not a stringent requirement.

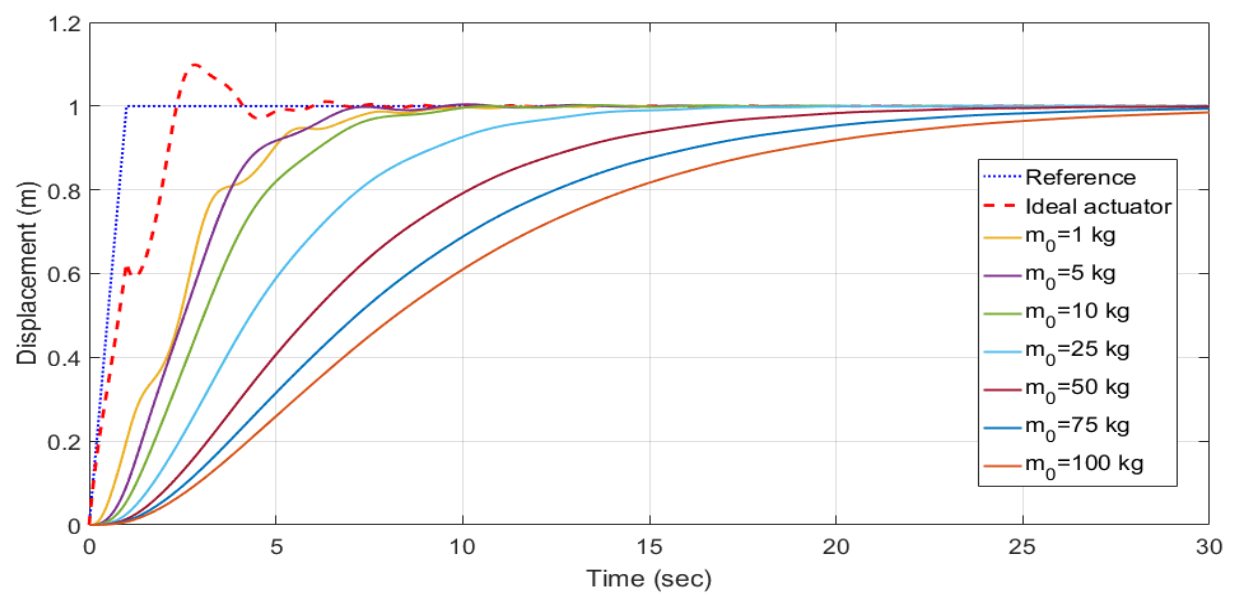

Fig.6. Responses of the non-ideal actuator applied to control the system shown in Fig. 4 for a range of $m_{0}$ values (or inertia of the real actuator) using the new WBC formulation compared with that of an ideal actuator (shown by dash line) 
Figure 7 shows the response of all components of the system used in Fig. 5 and for the same maneuver using the apparently appropriate value of $k_{0}=50 \mathrm{~N} / \mathrm{m}$. Compared to a system with an ideal actuator, the system is subjected to more time delay to settle, which is not surprising in view of the large inertia of the actuator as well as high ratio of $m_{0} / m_{1}$. But the response is stable, reasonably rapid, and settles precisely at target. As explained above, the choice of $k_{0}$, the notional spring constant, has a direct effect on the force levels. The force levels are also affected by the reference input waveform (the level of acceleration requested of the system), the total inertia of the system, and the degree of flexibility. But other things being equal, the greater $k_{0}$, the larger the force. In practice the source of the force will be limited, with some maximum value. If this value is reached, the actuator force will saturate. But once again, because WBC is based not on the requested force, but on the actual force achieved, the measured waves will automatically adjust and will still achieve position control and active vibration damping. The only degradation will be that the process may be affected by time delay. In the work above however, saturation of the force input was not studied in detail.

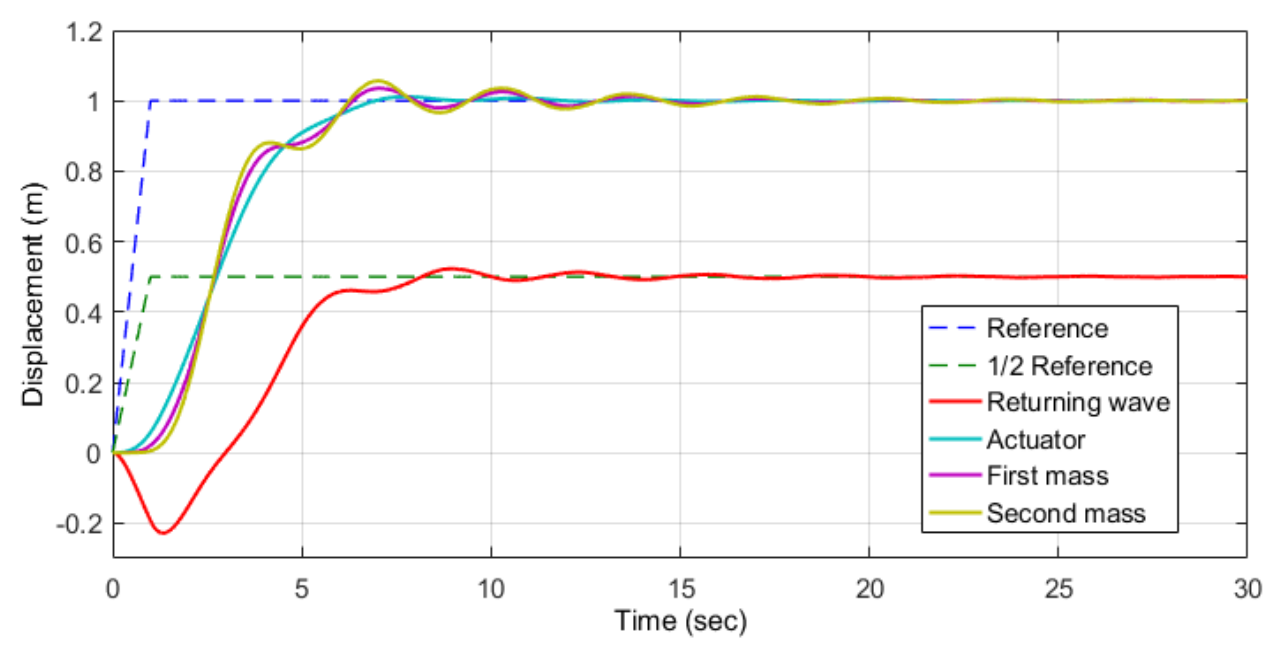

Fig. 7. Using the new WBC formulation for the two-mass system shown in Fig. 4 when $k 0=50 \mathrm{~N} / \mathrm{m}$

While presented here for lumped systems, the wave-based control technique works even better for completely distributed (continuous) systems, in which, because of finite time delays in wave propagation, wave concepts behind the proposed controller are unambiguous and more easily measured. This issue has been investigated well in a study presented in [21] in which a transfer function for the angular displacement of a flexible, cylindrical shaft around its axis was developed. The wave motion is manifested by the time delays in the developed transfer function where the number of delays indicate the possible routes the wave may take to travel from the actuator to a measurement point. The WBC (primarily designed for lumped systems) was first reformulated and adjusted to this continuous system where the new formulation contains the terms that are proportional to current and delayed versions of the reference and the measurement. It was finally shown that, for the flexible shaft, the WBC law is actually the same as another investigated method called Absolute Vibration Suppression (AVS) while their algorithms seem completely different. Illustrative examples in this study showed the two methods share the same response and perform successfully in removing vibration from the system. 
In this work, by choosing the number and value of the lumped parameters shown in Fig.1, the dominant modal shapes and frequencies of a continuous system can be matched and thereby its essential dynamics and control also investigated. If the system has distributed components or is predominantly distributed in nature rather than lumped, again there are no new control issues to grapple with.

\section{A challenging application: Slewing control of spacecraft}

This section extends the application of new WBC formulation from the relatively simple rectilinear system to the case of 2-D motion of a model of a satellite with large flexible appendages, shown schematically in Fig. 2, and in more detail in the upper part of Fig.8. Arrays of masses and springs are used to model two appendages, which may or may not have the same dynamics, that is, they may have different lengths, masses and springs. A lumped model, comprising a grid of masses, springs and dampers, is chosen mainly for ease of modelling. Each mass is subjected to forces in the springs connected to surrounding masses. It should be noted that such a lumped model can capture many important dynamic features of real systems, as well as presenting the flexible control challenge. Some systems are inherently lumped, such as solar panel arrays with light, flexible hinges, making a lumped model very appropriate.

On the spacecraft, the actuators providing torque and linear thrust will be on board the main body. As noted, these actuators must control the motion of the main body while simultaneously absorbing the vibrations of the appendages. The entire system is floating, with no grounded actuation system. The new version of wave-based control will be now adapted and tested for this case.

\subsection{Adapting the new development to the satellite model}

Typically the main satellite body (represented in the top centre of Fig.8) with its rocket motor or thrusters, reaction wheels, communication systems, fuel, batteries, instrumentation, and so on, will have much larger inertia than the appendages. If it is thought of as the "actuator", it is clearly an actuator of larger mass and moments of inertia in comparison with the rest of the system, which it must also control. Here we consider control of translation in two planar directions $(x$ and $y$ ) and of rotation $(\theta)$. There should therefore be three reference input signals $(x, y$ and $\theta)$ and correspondingly the relevant actuators need two input forces and one input torque to provide all possible motions in the plane. It is assumed that there are rockets (or chemical thrusters) to provide the forces, and reaction wheels (or pairs of thrusters, or even magnet torquers) to provide torques, each directly controllable.

The schematic of the proposed control technique is shown in Fig.8. The complete controller is designed simply as three parallel WBC controllers, considered above for Fig.4, each controlling one of the degrees of freedom of the main spacecraft's motion. The displacements of three ideal, notional actuators (whose positions are shown by $x_{-l}, y_{-l}$ and $\theta_{-1}$ ) are 
used to determine the input forces and torque, $f_{c x}, f_{c y}$ and $M_{c}$ to be applied to the spacecraft body, of total mass and inertia $m_{0}$ and $I_{0}$. The inputs to the notional actuator are $c_{x}, c_{y}$ and $c_{\theta}$. The forces and torque are determined using two notional linear springs of stiffness $k_{0 x}$ and $k_{0 y}$ and one notional torsion spring of stiffness $k_{t}$. The values $m_{-1}$ and $I_{-l}$ for the imaginary actuator can be assumed to be unity (because, being notional, its dynamics are also notional). The flexible appendages here are modelled by beam-like arrays of lumped masses, springs and dampers (the latter not shown, and frequently set to zero), whose parameters can easily be adjusted to represent different appendage dynamics.

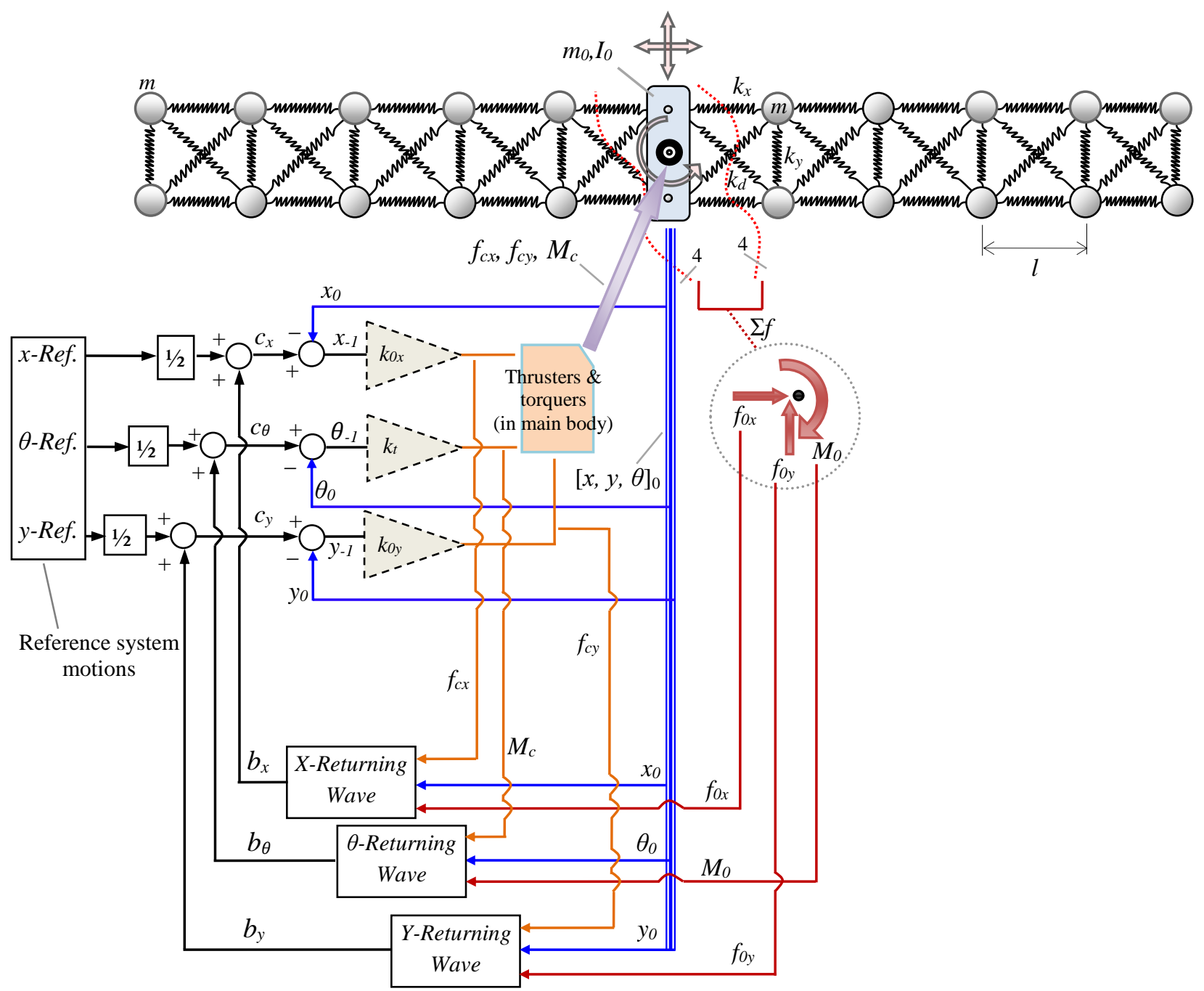

Fig. 8. Schematic explanation of the adapted new WBC formulation for planar satellite

When the main satellite body moves, it experiences four spring reaction forces on each side. The resultant of these reactions denoted by $f_{0 x}$ and $f_{0 y}$ in Fig. 8 play the same role as the $f_{0}$ in Fig.4. In addition $M_{0}$ is the resultant torque caused by the eight spring force acting back on satellite body even if the rotation reference (angular input) is zero. This reaction torque is very important for suppressing oscillations. The moment of the forces is calculated about an axis through the mass center of the system. (For reasons we omit here, choosing another moment axis will generally lead to final attitude errors at steady state). In practice, once again the controller sets the launch waves to be half the desired, or reference motions 
$\left(x_{r e f}, y_{\text {ref }}\right.$ or $\left.\theta_{\text {ref }}\right)$. Simultaneously it measures the returning waves and adds these to the launch waves to determine the desired displacements of the notional actuators, giving

$$
C_{q}=\frac{1}{2} q_{r e f}+b_{q}
$$

where $b_{q}(q=x, y$, and $\theta)$ are given by Eqs. (14). These desired motions are then converted to forces by multiplying the extensions of these notional springs by notional spring stiffness $\left(k_{0 x}, k_{0 y}\right.$ and $\left.k_{t}\right)$. One may think of the spring stiffness simply as a proportional constant in a P controller, although here it is not applied to an error as in conventional PID controllers.

The forces and torque are therefore specified as

$$
\begin{gathered}
f_{c x}=k_{0 x}\left(c_{x}-x_{0}\right) \\
f_{c y}=k_{0 y}\left(c_{y}-y_{0}\right) \\
M_{c}=k_{t}\left(c_{\theta}-\theta_{0}\right)
\end{gathered}
$$

As indicated in Fig.8, the control strategy for each of the three possible component motions is the same as for the rectilinear case of Fig.4. The three returning waves, $b$, are added to half the reference, to provide active vibration damping while repositioning the system at target. According to the measurements required for new formulation in WBC, given by Eq. (11) for a rectilinear system, the returning waves shown in Fig.8 are

$$
\begin{aligned}
& b_{x}=\frac{1}{2}\left(x_{0}-\frac{1}{Z_{c x}} \int_{0}^{t} f_{c x}(t) d t+\frac{1}{Z_{0 x}} \int_{0}^{t} f_{0 x}(t) d t\right) \\
& b_{y}=\frac{1}{2}\left(y_{0}-\frac{1}{Z_{c y}} \int_{0}^{t} f_{c y}(t) d t+\frac{1}{Z_{0 y}} \int_{0}^{t} f_{0 y}(t) d t\right) \\
& b_{\theta}=\frac{1}{2}\left(\theta_{0}-\frac{1}{Z_{c t}} \int_{0}^{t} M_{c}(t) d t+\frac{1}{Z_{0 t}} \int_{0}^{t} M_{0}(t) d t\right)
\end{aligned}
$$

where $x_{0}, y_{0}$, and $\theta_{0}$ are the actual motions of the satellite body, or actual translational and rotational displacement of the thruster, in the direction of reference coordinates $x, y$ and $\theta$, respectively. They are calculated and updated in each time step using the equations of motion for the satellite rigid body according to the Newton's $2^{\text {nd }}$ law of motion $(F=m a)$, by double integration of the relevant accelerations in

$$
\begin{aligned}
& m_{0} \ddot{x}_{0}=f_{c x}-f_{0 x} \\
& m_{0} \ddot{y}_{0}=f_{c y}-f_{0 y} \\
& I_{0} \ddot{\theta}_{0}=M_{c}-M_{0}
\end{aligned}
$$


For example, to work out $x_{0}$, the actual motion of the thruser in the direction of coordinate $x$, Eq. (15a) would be rearranged and used as $\ddot{x}_{0}=\frac{f_{c x}-f_{0 x}}{m_{0}}$ and integrated twice over time in each time iteration where $f_{0 x}$ is the resultant of elastic reaction forces in direction $x$ imposed by the attached appendages and $f_{c x}$, the input force to the thruster, is determined by Eq. (13a).

As before, the Z, mechanical impedances, terms in Eqs. (14) can be chosen to fine-tune the responses [18]. Their valuesare not crucial. In the current case, for example, the impedances were chosen as $\mathrm{Z}_{\mathrm{cx}}=0.5 * \operatorname{sqrt}\left(k_{0 x} * m_{0}\right)$, $\mathrm{Z}_{0 \mathrm{x}}=1.0 * \operatorname{sqrt}\left(k_{x}^{*} m\right), \quad \mathrm{Z}_{\mathrm{cy}}=0.5 * \operatorname{sqrt}\left(k_{0 y} * m_{0}\right), \quad \mathrm{Z}_{0 \mathrm{y}}=1.0 * \operatorname{sqrt}\left(k_{y} * m\right), \quad \mathrm{Z}_{\mathrm{ct}}=0.5 * \operatorname{sqrt}\left(k_{t}^{*} I_{0}\right), \quad$ and $\quad \mathrm{Z}_{0 \mathrm{t}}=1.0 * \operatorname{sqrt}\left(k_{d} * m\right) . \quad \operatorname{The}$ impedances with subscript ' $c$ ' correspond to the mass and inertia of the satellite body $\left(m_{0}\right)$ and to the stiffness of notional springs, whereas those with subscript ' $O$ ' refer to the lumped mass and stiffness of the attached flexible system $(m)$.

\subsection{Model Asymmetry as an added control challenge}

If the appendages on a satellite body are symmetrical, as for example when it has two identical solar panel arrays on each side, the control challenge is halved. A control strategy that actively dampens one appendage will also dampen the other, automatically. The problem is clearly more severe if the attached flexible systems have different dynamics and so are not symmetrical. To check out how WBC might deal with this problem, the mass and stiffness values in the appendages in Fig. 8, as well as their lengths (number of masses and springs) were varied to create very different dynamic characteristics on each side of the satellite body.

It might be expected that the asymmetry would create significant problems for the control system, as the main satellite body is attempting to use a single motion to launch and absorb waves in very different flexible systems simultaneously. In fact, WBC proved to have a great ability to deal with unsymmetrical or non-uniform flexible systems without any need for modification of the controller, even with such a complicated spacecraft model. Some sample results will be illustrated in Section 4. The explanation would seem to be the same as in previous applications controlled by WBC. Even if the spacecraft fails to absorb returning waves properly when they first return to the main satellite body, provided it absorbs a good fraction of the wave, it simply reflects the remainder, which it then tries to absorb on its second return, and so on. So the entire system continues to absorb vibrations, even if not perfectly, while simultaneously settling at the target position, as before.

\section{Sample results}

The success for rectilinear control was shown in Section 2. The new version of WBC is now applied to a $2 \mathrm{D}$, multiappendage spacecraft model with 43 DOF (including the rigid, bar-like actuator) as depicted in Fig.8. As the first test, a planar manoeuvre of simultaneous $x$ and $y$ translation is requested, using absorbing of the returning waves in all three 
directly controlled variables calculated by Eqs. (14). In this test, the appendages have uniform masses and are symmetric, with parameters shown in Table 1.

Table 1

Physical values for the satellite model shown in Fig. 8.

\begin{tabular}{cccccc}
\hline$k_{x}(\mathrm{kN} / \mathrm{m})$ & $k_{y}(\mathrm{kN} / \mathrm{m})$ & $k_{d}(\mathrm{kN} / \mathrm{m})$ & $m(\mathrm{~kg})$ & $m_{0}(\mathrm{~kg})$ & $l(\mathrm{~m})$ \\
\hline 7 & 8 & 10 & 0.2 & 50 & 0.25 \\
\hline
\end{tabular}

In this sample set $k_{x}, k_{y}$ and $k_{d}$ are the spring stiffness in horizontal, vertical and diagonal directions respectively, $m$ the mass values of the point masses in the appendages, $l$ the gap length between point masses and $m_{0}$ the mass of the main satellite rigid body. The actuator has therefore been chosen to be 250 times as massive as the point masses, or 12.5 times more than the whole attached flexible system. Figure 9 shows the response. The references are $1 \mathrm{~m}$ displacement in the $y$ direction and $0.6 \mathrm{~m}$ in the $x$-direction, at different ramp rates. Considering the large ratio of actuator to system masses, the quick rise towards the target displacement as well as the short settling time of the two furthest points of the system (less than 5 seconds) in both directions is impressive.

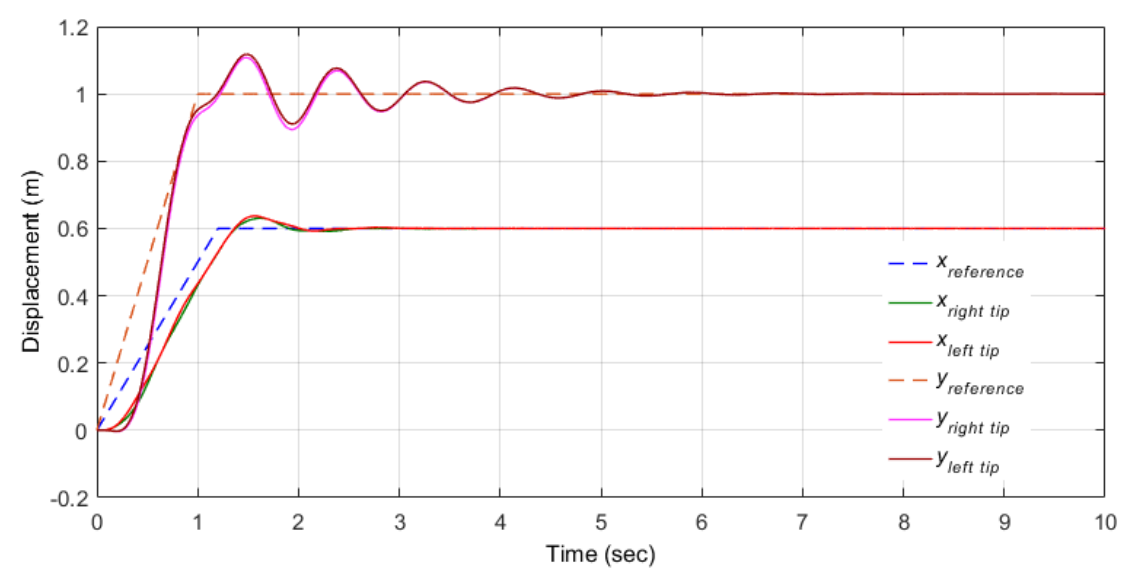

Fig. 9. WBC response of the satellite model for an $X-Y$ translation using the new WBC development

Figure 10 shows the magnitude and direction of the input forces applied to the spacecraft body during the maneuver shown in Fig. 9.

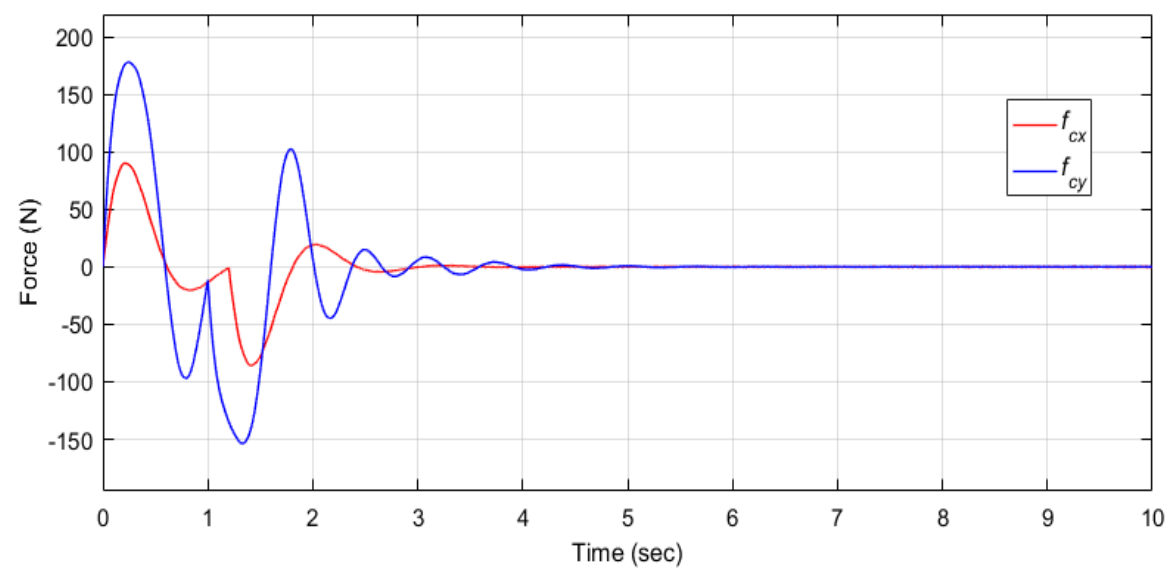

Fig. 10. The input forces applied to the spacecraft body in $x$ and $y$ directions during the maneuver depicted in Fig. 9. 
As a second test with the same parameters as in Table 1, the system is subjected to a translation and rotation simultaneously. The result is shown in Fig. 11 in which references are one radian, counter-clockwise rotation and 0.5 meter, $y$-direction movement. Despite the complexity of such a combined slewing and translation maneuver, the new WBC formulation proves well able to eliminate the remaining errors and to have only a small overshoot on first reaching the target. The system does display swaying or oscillatory behaviour on its way to the target and for a while after arrival. But some points should be made. First moving a flexible, longitudinal-extended system perpendicular to its longitudinal axis, while also imposing a large rotation, would clearly be expected to lead to considerable flexing of the system. Second, the graphs shown in Fig. 11 correspond to the tips of the two lateral appendages, which therefore display the greatest oscillations within the system. In addition, the large mass ratio of actuator to flexible system and of the very many degrees of freedom also contributes to the residual vibration after arrival at the target and the somewhat extended settling time.

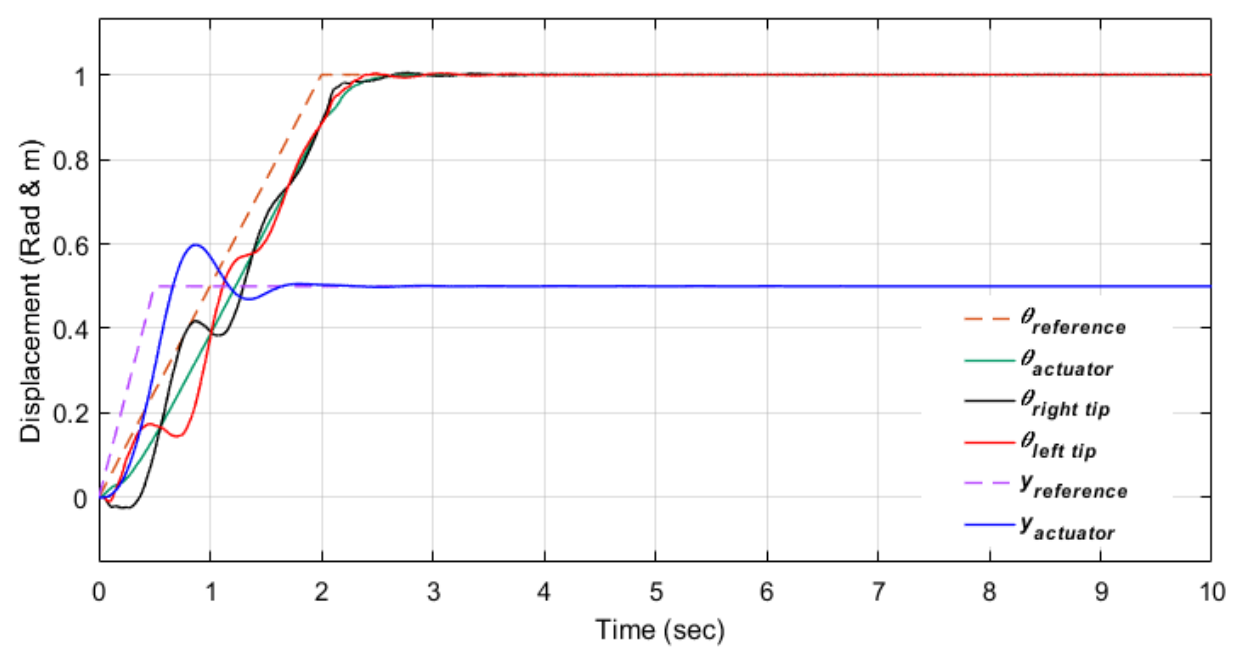

Fig. 11. WBC response of the satellite model using the new WBC development in a maneuver consisting of both translational and rotational motions

Figure 12 shows the magnitude and direction of the input torque applied to the spacecraft body change during the maneuver depicted in Fig. 11.

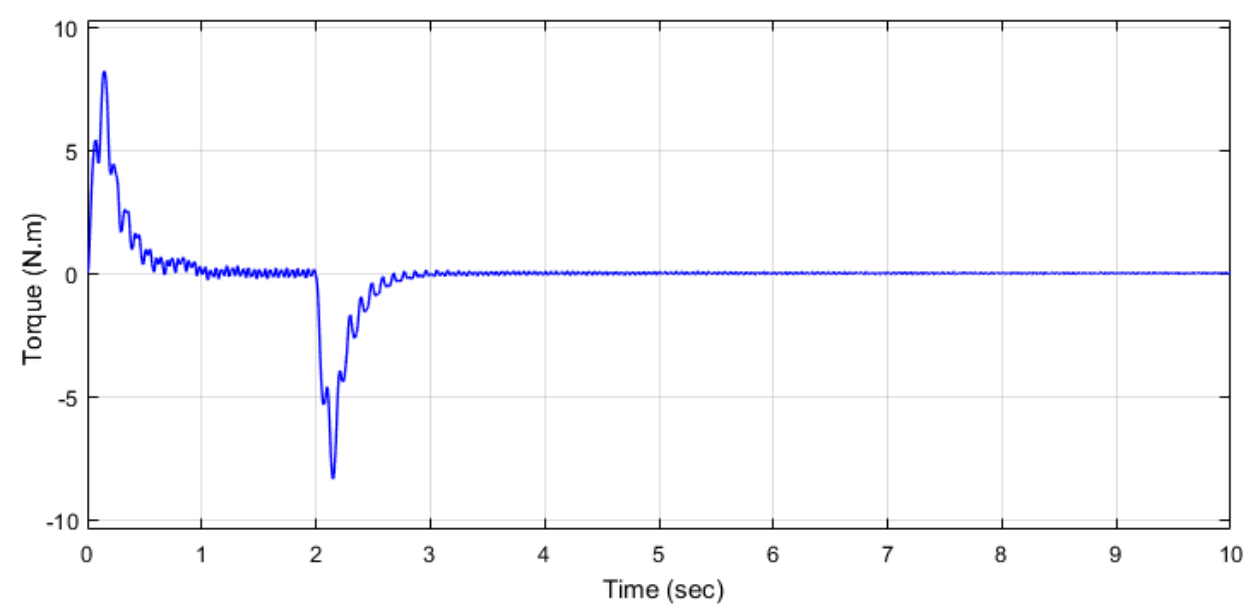

Fig. 12. The input torque applied to the spacecraft body during the maneuver depicted in Fig. 11. 
The test cases above show rest-to-rest manoeuvres with ramp inputs. To illustrate tracking of more continuously varying inputs, the spacecraft was requested to move from point A to B along a complicated planar trajectory, arbitrarily chosen, as shown in Fig.13, taking 10 seconds in total. Considering that the spacecraft is $2.5-\mathrm{m}$ long, the tracking can be seen to be good, with no major overshoot or steady-state errors, despite sharp corners and rapid motion along the track.

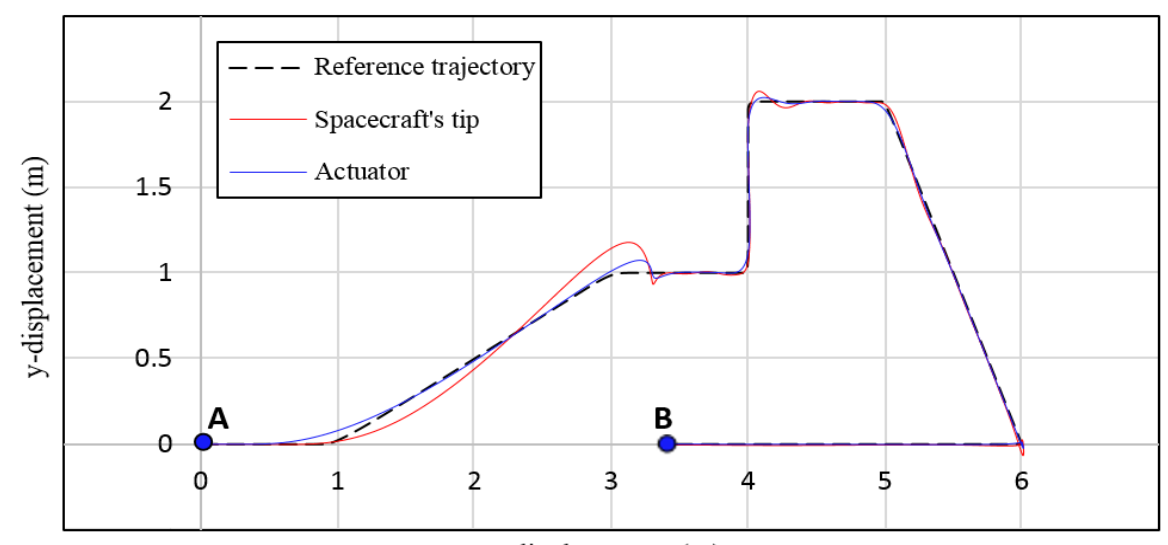

x-displacement (m)

Fig. 13. WBC response of the spacecraft with flexible panels to a trajectory tracking mission in a planar maneuver

Next, to test the robustness of the adapted new WBC formulation when the flexible appendages have different dynamics, the lateral panels were made different in two respects, namely length and mass. The left flexible panel of the model shown in Fig. 8 was given only two square segments, with overall length of $0.5 m$ whereas the right-hand panel kept its five segments, making it $1.25 \mathrm{~m}$ long. Furthermore, the value of each point mass in the left panel was set to $m_{L}=0.1 \mathrm{~kg}$ against masses of $m_{R}=0.5 \mathrm{~kg}$ on the right-hand side. So altogether the right panel becomes $(5 / 0.4=) 12.5$ times more massive and 2.5 times longer than the left side. This arrangement simulates two very different appendages attached to the actuator. The other parameters such as the mass of the actuator and the stiffness of the springs in the three directions are the same as in Table 1.

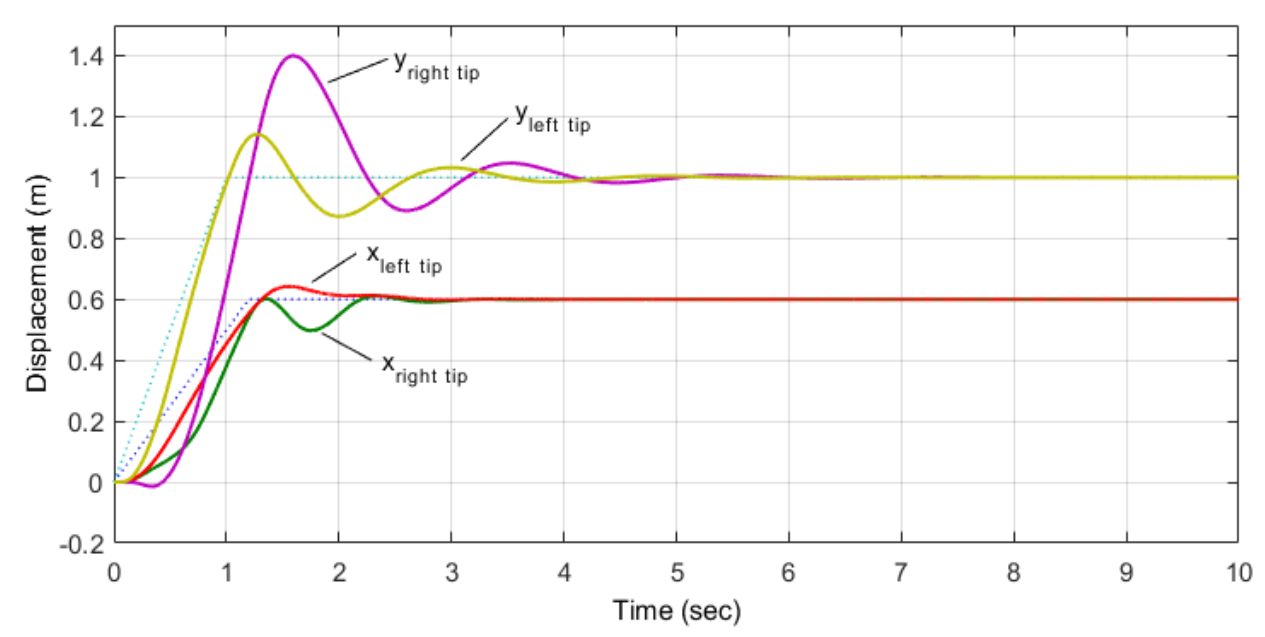

Fig. 14. WBC response of an asymmetric model of the 2D satellite model (Case 1) using the new adapted formulation 
Figure 14 shows how the furthest points on either side of the model respond to the same manoeuvre as in Fig.9. As can be seen, the response shows higher overshoot and longer transient time than in Fig.9 for the same points in the system. However, despite the more complex, unsymmetrical dynamics, and without any change or tuning of the controller from the previous tests, and in the absence of internal damping, WBC still very successfully moves the system from rest to rest, while rapidly absorbing the vibrations.

To provide more generality on the capability of the new development in WBC on controlling unsymmetrical systems, another asymmetric model of the satellite was implemented and run in which the two appendages are even more discrepant in length, mass, and also stiffness. In this model, one more square segment from the left flexible panel was removed which made its total length as $0.25 \mathrm{~m}$. Then the two remaining masses on the left panel were set to $m_{L}=0.15 \mathrm{~kg}$ against masses of $m_{R}=0.30 \mathrm{~kg}$ on the right-hand side while the spring constants interconnecting the left masses were reduced to $k_{x}=5 \mathrm{kN} / \mathrm{m}$, $k_{y}=6 \mathrm{kN} / \mathrm{m}$, and $k_{d}=7.5 \mathrm{kN} / \mathrm{m}$. So this time, the right panel becomes $(3 / 0.3=) 10$ times heavier, 5 times longer, and almost $\% 25$ stiffer than the left side. Figure 15 displays the displacement-time response of the actuator and a few different points on either side of the model undergoing a maneuver with displacement of $0.5 \mathrm{~m}$ in the $x$-direction and $1 \mathrm{~m}$ in the $y$-direction, at different ramp rates. As depicted in the figure, the new formulation of WBC still performs very well in providing simultaneous rest-to-rest motion control and vibration suppression of this complex model despite such inconsistent flexible appendages.

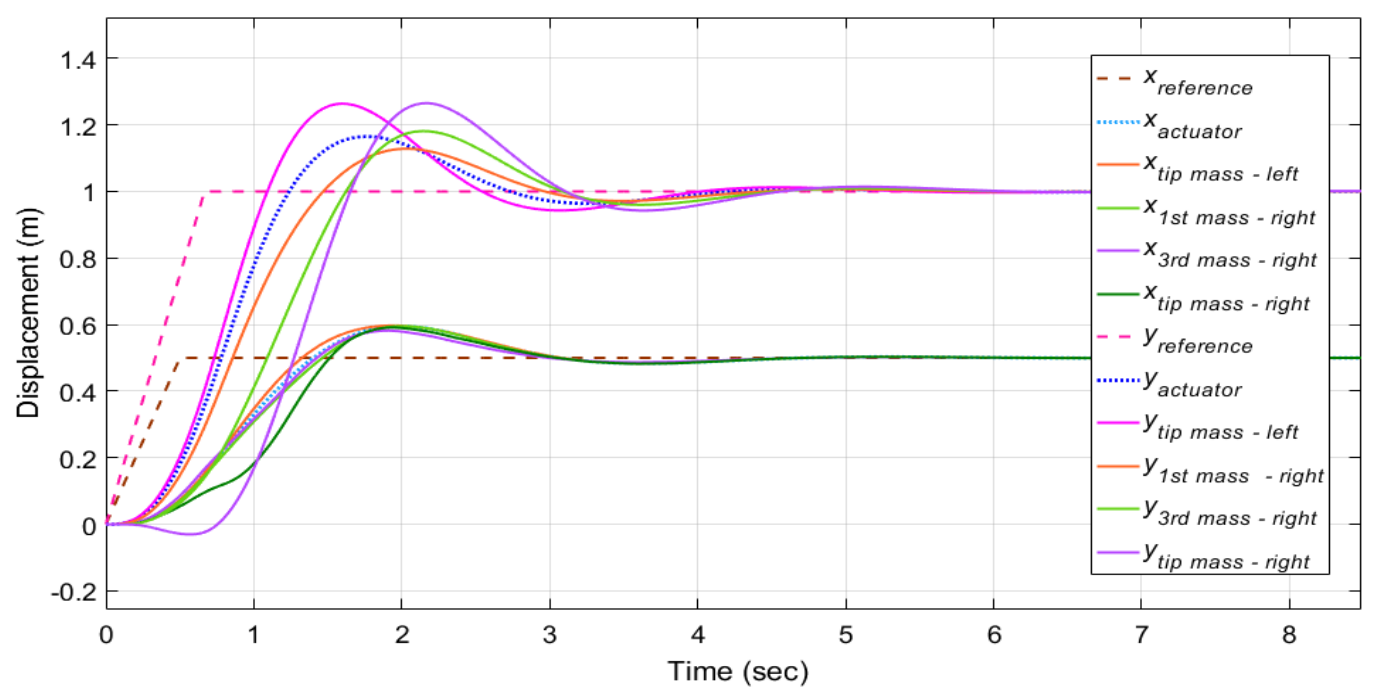

Fig. 15. WBC response of an asymmetric model of the 2D satellite model (Case 2) using the new adapted formulation

\section{Conclusions}

In this study, an evolution or adaptation of WBC has been developed in which the force acting on the actuator, $f_{c}$, is directly controlled, using measurements of this force and of the actuator motion, $x_{0}$. The motion variable, $c$, can be thought of as the position of a notional actuator which moves to produce the input force through a notional spring of stiffness, $k_{0}$, 
which then pushes the actuator mass. This dispenses with the actuator sub-controller, so that all control aspects are carried out by WBC itself. The robustness of the new WBC formulation was illustrated by the stability and successful control performance of a 2-mass flexible system with different mass ratios between the actuator and the system where the actuator reached up to fifty times heavier than the system masses $\left(m_{0} / m_{l}=50\right)$. In control terms, this could be considered as an actuator which was far from ideal. This control system works well for real (non-ideal) actuators associated with considerable inertia and time delay which are grounded, and for systems with "floating" actuators, such as spacecraft.

To assess this development in a challenging way, a 2D flexible model of a typical spacecraft driven by thrusters with multiple appendages was created. Apart from the novelty of an adapted version of this new formulation in WBC in controlling 2D lumped arrays, getting the system to control a few flexible appendages through only one actuator was achieved. In the proposed control scheme, the same as in the developed WBC control for the rectilinear system, there is still no need to use an actuator sub-controller. This new, adapted version of WBC retains all the principles of the wavebased idea and the associated benefits. It still makes all the measurements at the actuator, and still has no need for a perfect system model or detailed knowledge of the dynamical properties of the system. In addition, other challenging aspects associated with practical spacecraft control systems were investigated. The first was the ability to cope with any given trajectory as a reference path, whether it is translational, rotational or a combination of both, in spite of the complexity of the multiple attached flexible arrays of the many degrees of freedom. The next challenge was asymmetry in the model, making the dynamics of the system even more complicated. Different successful tests demonstrated that the $2 \mathrm{D}$ adapted version of the developed WBC formulation is able to control asymmetrical, multi-appendage systems, whether the nonuniformity is due to differences in masses and stiffness or in the lengths of the appendages. In all cases the proposed control strategy, in the absence of any kind of damping, drove the system quickly to the precise target displacement, linear or angular, while actively damping the vibrations.

\section{References}

[1] Mizutani K., Yatomi C., Inoue K., Active vibration control for flexible structures using a wave-absorbing control method, JSME International Journal Series C-Dynamics Control Robotics Design (1996), 39(3), 677-677.

[2] Jones R.W., Mace B.R., Mei C., Large Structure Vibration Suppression Using Distributed Wave Control, IFAC Proceedings Volumes, Vol. 32, Issue 2, July 1999, 8112-8117.

[3] O'Connor W.J., Lang D., Position control of flexible robot arms using mechanical waves, ASME Journal of Dynamic Systems, Measurement, and Control, 120 (3), 1998, 334-339.

[4] O’Connor W.J., Ramos F., McKeown D.J., Feliu V., Wave-based control of non-linear flexible mechanical systems, Nonlinear Dynamics, Springer, 2009, vol. 57, No 1-2, 113-123. 
[5] O’Connor W.J., McKeown D.J., Time-optimal control of flexible robots made robust through wave-based feedback, ASME J Dynamic Systems, Measurement \& Control, (2011), vol.133, Issue 1.

[6] Langmajer M., Schlegel M., Setka V., Stability Analysis of Wave Based Control: Practical Aspects, in Proceedings of the 21st International Conference on Process Control (PC), June 2017, ŠtrbskéPleso, Slovakia.

[7] Valášek M., Neusser Z., Gordon T., Wave-based control for intelligent longitudinal traffic column, in Proceedings of the 24th Symposium of the International Association for Vehicle System Dynamics (2016), Graz, Austria, 17-21 August.

[8] Habibi H. \& O’Connor W.J., Payload motion control of rotary gantry and luffing cranes using mechanical wave concepts, Transactions of the Institute of Measurement and Control, 2017, Vol. 39(11), pp. 1649-1662.

[9] Halevi Y., Control of flexible structures governed by the wave equation using infinite dimension transfer functions, ASME Journal of Dynamic Systems, Measurement \& Control, (2005), 127, 579-588.

[10] Sirota L., Halevi Y., Free response and absolute vibration suppression of second order flexible structures-the traveling wave approach, ASME J. Vib. Acoust., (2010), 132, p. 031008.

[11] Fan Y., Collet M., Ichchou M., Li L., Bareille O., Dimitrijevic Z., A wave-based design of semi active piezoelectric composites for broadband vibration control, Smart Materials and Structures (2016), 25(5), P. 055032.

[12] Zhou J., Zhang K., and Hu G., Wave-Based Control of a Crane System with Complex Loads, Journal of Dynamic Systems, Measurement, and Control, Vol. 139 (8), 081016, June 2017.

[13] Khalilpour S.A., Khorrambakht R., Taghirad H.D., Cardou P., "Wave Based Control of A Deployable Cable Driven Robot", Robotics and Mechatronics (IcRoM) 2018 6th RSI International Conference on, pp. 166-171, 2018.

[14] Khalilpour S.A., Taghirad H.D., Habibi H., "Wave-based Control of Suspended Cable Driven Parallel Manipulators", In proceeding of the 5th IEEE International Conference on Control”, Instrumentation and Automation (ICCIA2017), 21-23 November 2017, Shiraz, Iran.

[15] Liu Y., Zhang K., Zhang W-Z., and Meng X-Y., Wave-based vibration control of large cable net structures, Wave Motion, Volume 77, March 2018, Pages 139-155.

[16] McKeown D.J., Wave based Control of Elastic Mechanical Systems, Ph.D. Thesis, Department of Mechanical Engineering, University College Dublin, Ireland, 2009.

[17] O’Connor W.J., A Gantry Crane Problem Solved, ASME Journal of Dynamic Systems, Measurement, and Control, 2003, 125, 569-576.

[18] O’Connor W.J., Habibi H., Gantry crane control of a double-pendulum, distributed-mass load, using mechanical wave concepts, Mech. Sci., 2013, 4, 251-261.

[19] Liu G.P., Daley S., Optimal-tuning PID controller design in the frequency domain with application to a rotary hydraulic syste, Control Engineering Practice, 2001, 7, 1185-1194.

[20] Ang K.H., Chong G.C.Y., Li Y., PID control system analysis, design, and technology, IEEE Trans Control Systems Tech., 2005, 13(4), 559-576.

[21] Peled I., O’Connor W.J., and Halevi Y., On the relationship between wave based control, absolute vibration suppression and input shaping, Mechanical Systems and Signal Processing 39 (2013) 80-90. 